Document downloaded from:

http://hdl.handle.net/10251/48362

This paper must be cited as:

Fages, E.; Cano, M. A.; Girones, S.; et ál..(2013). The use of wet-laid techniques to obtain flax nonwovens with different thermoplastic binding fibers for technical insulation applications. Textile Research Journal. 83(4):426-437. doi:10.1177/0040517512454183.

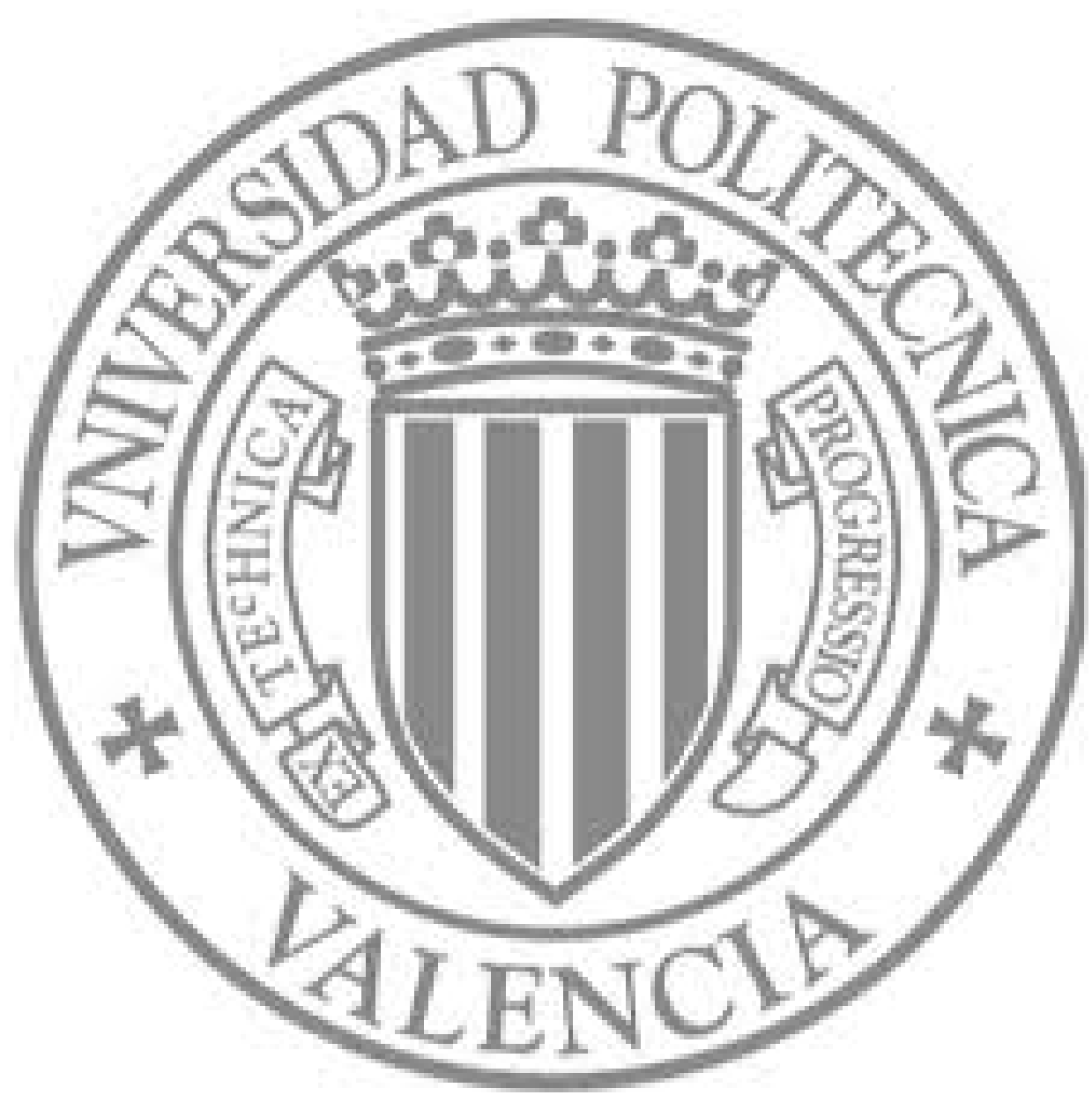

The final publication is available at

http://dx.doi.org/10.1177/0040517512454183

Copyright SAGE Publications (UK and US) 


\title{
"The use of wet-laid techniques to obtain flax nonwovens with different thermoplastic binding fibers for technical insulation applications"
}

\begin{abstract}
In this work, the wet-laid technique has been used to obtain flax nonwovens thermally-bonded with different contents of polyvinyl alcohol (PVA) and bicomponent polyamide 6/copolyamide (PA6/CoPA) fibers in the 10-30 wt. \% range. Morphology of nonwovens has been studied by scanning electron microscopy (SEM) to evaluate formation of interlock points through melted polymer and flax fibers. Main physical properties (thickness and surface mass) have been determined by standardized tests. Tensile strength and elongation at break have been determined with standard test procedures on longitudinal (preferential deposition direction in hydroformer station) and transversal directions to evaluate anisotropic behavior of nonwovens. The sound absorption properties of stacked sheets of flax:PVA and flax:PA6/CoPA nonwovens have been evaluated with an impedance tube by determining the absorption coefficient in terms of the sound frequency. In addition, thermal insulating properties of individual nonwovens have been tested with the heat flow meter method. Mechanical characterization shows slight anisotropy since higher tensile strength values are obtained in the longitudinal (preferential) direction. The absorption coefficient is interesting in the medium frequencies range and relatively low thermal conductivity and thermal resistance values are obtained with these nonwovens. By taking into account these features, these nonwoven substrates could find interesting applications as sound absorbers and/or thermal insulation materials in technical applications.
\end{abstract}

Keywords: fiber, yarn, fabric formation; environmental sustainability; properties; materials 


\section{Introduction.}

In the last years, a growing interest on the use of biobased materials or materials from renewable resources has been detected. This interest has also arrived to technical sectors such as building, automotive, transportation, etc. industries due to increasing environmental concerns, potential biodegradability and use of overall eco-friendly materials. Some of the requirements of these technical sectors are focused on comfort and this means that base materials must provide good insulation behavior, both thermal and acoustic ${ }^{1,2}$. Mineral and synthetic polymer wools are interesting substrates which are able to offer easy handling and good insulation properties due to the internal structure, therefore their use in building industry and other technical sectors has been generalized ${ }^{3-7}$; nevertheless, new materials from renewable resources are been demanded since they could offer similar behavior than traditional heat and sound absorbers, so that, important efforts on the development of natural (plant or animal derived) materials are being done.

In the last decade, a growing use of natural fibers such as hemp, kenaf, pineapple, abaca, flax, coir, etc. and other biobased fibrous components in the form of nonwovens or short fibers has been detected and research on this field is increasing ${ }^{8-10}$. These natural fibers could find potential uses as technical textiles due to excellent balanced properties ${ }^{11}$. The potential of natural fiber nonwovens opens a wide variety of new applications in the textile industry, for example as biodegradable wipes ${ }^{12}$ and also in other technical sectors such as automotive ${ }^{7,13,14}$. Nonwoven structures can be used in a flexible form as thermal and acoustic insulation materials in automotive interior parts as lining materials due to particular shape and internal structure of natural fibers ${ }^{15-}$

18. In addition, hybrid nonwovens with natural fibers and small amounts of binding 
fibers can lead to obtaining base materials for composite production by hot-press molding as rigid interior panels in automotive and transportation industries with balanced mechanical performance and acoustic and thermal insulation behavior ${ }^{19}$. In this field, interesting results have been obtained by using bicomponent fibers in which, the sheath can be melted by temperature to promote a homogeneous matrix thus leading to composites with interesting applications in technical sectors ${ }^{20-22}$. The use of nonwovens is also generalized as filtering media and new substrates based on natural fibers are being developed ${ }^{23,24}$.

The wet-laid process, a widely used process in the paper industry, represents an interesting technology to obtain nonwoven structures based on different base components both raw and waste materials. Short fibers from microns up to 15-20 mm can serve as base materials for nonwoven production in a continuous way ${ }^{25}$. Furthermore, hybrid nonwoven structures can be obtained by combining different fibers so that it is possible to mix a base natural fiber with a binding fiber to provide cohesion after a thermo-bonding process ${ }^{26-28}$. The wet-laid process, which uses highly diluted fiber-water dispersions, is an eco-friendly process since, although it consumes high water amounts, all the water is recirculated since it only acts as the fiber carrier component so that, almost all water is recovered in the hydroformer station in which nonwoven formation occurs ${ }^{29}$.

The main aim of this work is to determine the potential of flax-based nonwovens obtained by the wet-laid process as candidate materials for thermal and acoustic insulation applications. To provide cohesion on nonwovens, different thermoplastic binder fibers have been used: polyvinyl alcohol (PVA) as fully biodegradable thermoplastic fiber and a typical binder bicomponent polyamide6/copolyamide (PA6/CoPA) fiber. Optimum processing conditions in the-wet laid process are 
determined. The morphology of nonwovens is characterized by scanning electron microscopy (SEM). The main physical properties such as thickness and surface mass are determined by standardized tests. In addition, mechanical performance on longitudinal (preferential formation direction in the hydroformer station) and transversal directions is evaluated by determining tensile strength and elongation at break in terms of the binder fiber type and content. The potential of these nonwovens as sound absorber and/or thermal insulation materials is determined with standardized tests using an impedance tube to determine the absorption coefficient and a heat flow meter apparatus to determine the thermal conductivity and thermal resistance.

\section{Experimental.}

\subsection{Materials.}

Commercial flax fibers F 513/6 supplied by STW Fibres were used as base fibres for nonwoven formation with technical flax fibres (50-100 $\mu \mathrm{m}$ thickness) and elementary fibres with a thickness comprised between 10-20 $\mu \mathrm{m}$. General properties of flax fibres are summarized in Table 1.

\section{Table 1}

The thermo-bonding process was carried out with different thermoplastic fibres: polyvinyl alcohol (PVA) and a bicomponent fibre with a polyamide 6 (PA6) core sheathed by a copolyamide (CoPA).

PVA fibres, commercial grade PVA 401/6, were supplied by STW Fibres. The degradation temperature is above $240{ }^{\circ} \mathrm{C}$ and the softening temperature is close to 120 
${ }^{\circ} \mathrm{C}$. The highly hydrophilic nature of these fibres enables good dispersion in aqueous media. With regard to the bicomponent fibre, a commercial grade Grilon ${ }^{\circledR}$ BA 140 supplied by EMS-GRILTECH was used. The core (50 wt. \%) consists on polyamide 6 (PA6) polymer with a melt point of $222^{\circ} \mathrm{C}$ while the sheath (50 wt. \%) is composed of a low melt point $\left(135^{\circ} \mathrm{C}\right)$ copolyamide $(\mathrm{CoPA})$.

\subsection{Formation of non-woven flax-thermoplastic fiber substrates by wet-laid process.}

\subsubsection{Weighting and fibre separation.}

In a first stage, the appropriate fibre amounts are weighted in order to obtain different flax/thermoplastic binder ratios: 90/10, 80/20 and 70/30.

Initially, fibres are separated in water by using a high shear pulper (PILL NASSVLIESTECHNIK GmbH WET-LAID NONWOVEN TECHNOLOGY) with a total capacity of $35 \mathrm{~L}$. The fibre concentration in the water dispersion is maintained at $10 \mathrm{~g} \mathrm{~L}^{-}$

1 and the mixing process has been carried out with strong agitation at $2300 \mathrm{rpm}$ for 10 $\min$.

\subsubsection{Maintenance of fibre dispersion in water.}

After the initial separation and subsequent dispersion in water, the water-flax dispersion is dropped into a dispersion tank with total capacity $1200 \mathrm{~L}$ in which, the flax dispersion is diluted to a concentration of $1 \mathrm{~g} \mathrm{~L}^{-1}$ and it is subjected to less vigorous agitation at $170 \mathrm{rpm}$ to maintain good fibre dispersion for a total time of $15 \mathrm{~min}$.

\subsubsection{Nonwoven formation.}

Once fibres are appropriately dispersed in aqueous solution the water-fibre mixture is moved to the hydroformer station by using hydraulic pumps. The 
hydroformer station has been supplied by PILL NASSVLIESTECHNIK GmbH and it is constructed in stainless steel with different polycarbonate windows to observe the process evolution. The maximum width is $510 \mathrm{~mm}$, the take-off angle is $20^{\circ}$ and the conveyor speed can vary between 1 and $10 \mathrm{~m} \mathrm{~min}^{-1}$. Two different tanks are used to ensure optimum dispersion conditions; when one of these two tanks is used, the other is empty and vice versa. The water-fibre dispersion is pumped to the hydroformer station as observed in Scheme 1, but before reaching to the forming strip, the water-fibre dispersion is diluted once again up to a final concentration of $0.33 \mathrm{~g} \mathrm{~L}^{-1}$. Then the water-fibre dispersion is dropped onto the porous forming strip which acts as a filter media in which water is removed by vacuum and fibres are deposited.

\section{Scheme 1}

\subsubsection{Nonwoven consolidation}

Consolidation of flax nonwovens has been carried out by a thermo-bonding process in two stages; a first drying stage with hot air followed by a second thermobonding process in a hot calender. The drying module (SDT-600) and the calendering equipment (CL-600) have been supplied by TALLERES TACOME S.A. The main parameters for the drying and calendering of nonwoven substrates are summarized in Table 2. Finally, nonwovens are rolled in a roller module (EN-600) supplied by TALLERES TACOME S.A.

Table 2 


\subsection{Morphology characterization by SEM.}

Surface morphology of PVA and PA6/CoPA thermo-bonded flax nonwovens has been evaluated by scanning electron microscopy with a Quanta 200SEM at a voltage of $25 \mathrm{kV}$. Different images at 200x and 400x have been recorded without previous sputtering.

\subsection{Determination of thickness and surface mass.}

Thickness of nonwovens has been measured with a Sodemat thickness apparatus with a test pressure of $0.5 \mathrm{KPa}$, following the guidelines of the ISO 9073-2:1997. Surface mass was measured on $1 \mathrm{dm}^{2}$ shapes obtained by cutting nonwovens with a circular die mod. 1.333 RS supplied by Horvecal S.A. Five different samples have been cut and weighed in a precision balance model GR-200-EC by A\&D Instruments Ltd. Temperature and relative humidity have been maintained at $20 \pm 2{ }^{\circ} \mathrm{C}$ and $65 \pm 4 \%$ respectively for both tests.

\subsection{Characterization of mechanical properties.}

Tensile properties (both longitudinal and transversal) of nonwovens have been determined by following the guidelines of the ISO 29073-3:1993 with an Instron dynamometer model 4501 (Instron, Barcelona, Spain). The clamp distance was set to $200 \mathrm{~mm}$ and the crosshead rate was $100 \mathrm{~mm} \mathrm{~min}^{-1}$. At least five samples were tested and average values for tensile strength and elongation at break were calculated. Temperature and relative humidity have been maintained at $20 \pm 2{ }^{\circ} \mathrm{C}$ and $65 \pm 4 \%$ respectively. 


\subsection{Thermal and acoustic insulation characterization.}

The thermal insulation properties have been evaluated by determining the thermal conductivity and thermal resistance according to the guidelines of the ISO 8301:1991 standard with a heat flow meter apparatus HFM 436 Lambda supplied by

NETZSCH. In this method, samples sizing $30 \times 30 \mathrm{~cm}^{2}$ are placed between a hot and a cold plate and the heat flow created by the well-defined temperature difference is measured with a heat flux sensor thus allowing the calculation of both thermal conductivity and thermal resistance.

Acoustic insulation properties have been evaluated on nonwovens with a flax:binder fiber ratio of 80:20, by measuring the absorption coefficient of 12 stacked nonwoven substrates by following the guidelines of the ISO 10534-2:2002 standard with an impedance tube kit Type 4206 supplied by Brüel\&Haer. A double channel Symphonie analyzer with FFT has been used with $1 / 2$ in and $1 / 4$ in microphones. The experimental frequency has varied in the $50-6400 \mathrm{~Hz}$ with a step of $2 \mathrm{~Hz}$.

\section{Results and discussion.}

\subsection{Morphology of flax nonwovens thermo-bonded with PVA and COPA fibres.}

SEM images of flax nonwovens thermally bonded with different amounts of PVA fibers do not show clear melted PVA areas (Fig. 1). As we have described previously, the softening point of PVA is close to $120^{\circ} \mathrm{C}$. The thermal-bonding process has been carried out at $195^{\circ} \mathrm{C}$ but even the use of this relatively high drying temperature is not enough to fully melt PVA fibers to embed flax fibers. This phenomenon could be related to the fact that PVA fibers absorb water (up to 4-6 wt. \%) so that, it is necessary the use of higher temperatures or longer drying times to fully melt PVA polymer. This morphology would play a key role on overall mechanical performance of flax:PVA 
nonwovens; but even in this case, good thermal and acoustic insulation properties can be achieved since softened PVA fibers can interact with some flax fibers.

\section{Figure 1}

With regard to flax nonwovens with PA6/CoPA bicomponent fibers as binder (Fig. 2), we can clearly detect a decrease in porosity as the total amount of PA6/CoPA fiber increases. We can also observe the typical shape and geometry of individual flax fibers as they show a wide range of variability in diameter between $10-500 \mu \mathrm{m}$. As we have described before, the sheath is based on a low melt point copolyamide (CoPA) with a melt point close to $135^{\circ} \mathrm{C}$; so that, the use of temperatures close to $195^{\circ} \mathrm{C}$ during drying and subsequent calendering is enough to fully melt the sheath which represents 50 wt. $\%$ of total binder fiber. So that, the real binder fiber content (CoPA) is around 5, 10 and $15 \mathrm{wt}$ \%, corresponding to total PA6/CoPA contents of 10, 20 and 30 wt. \% on flax nonwovens. We can clearly observe presence of binding areas characterized by partially embedded flax fibers. As CoPA melts it moves over individual flax fibers thus embedding them. These melted areas are responsible for formation of interlock points which can provide higher mechanical resistance than previously described flax:PVA nonwovens.

\section{Figure 2}

The binding structure of thermally-bonded nonwovens highly depends on the heat transfer method as well as the web structure and the nature of the binding fiber. By using thermal calendering, heat transfer is achieved by conduction phenomena; this 
produces the melt of the CoPA sheath on PA6/CoPA fibers. The melted polymer which covers the PA6 core fiber can also partially embed flax fibers due to the high mobility of melted polymer and this embedded fibers form flax-flax and flax-PA6 interlock points which will have a positive effect on mechanical resistance of nonwovens. As a result, flax:PA6/CoPA nonwovens are characterized by a more stable structure since the core remains solid during the drying-calendering process while the sheath melts at $135^{\circ} \mathrm{C}$. Then, the melted sheath acts as fusible adhesive and it can be distributed very finely throughout the supporting PA6 and flax fibers and the solid PA6 core provides strong support for nonwovens both during and after bonding.

As the real binding component (CoPA) represents only 5, 10 and 15 wt. \% it is homogeneously distributed over PA6 supporting fibers but in addition, the melted CoPA polymer can adhere to nearby flax fibers, thus acting as a bridge to form flax-flax and flax-PA6 interlock points. Nonwovens with a PA6/CoPA content of 30 wt. \% (15 wt. \% CoPA binder) are characterized by a homogeneous flax:PA6 nonwoven structure homogeneously embedded in a CoPA matrix (Fig. 2e and 2f).

\subsection{Physical and mechanical properties of flax nonwovens thermally-bonded with different thermoplastic fibers.}

With regard to flax:PVA nonwovens, the surface mass is close to $400 \mathrm{~g} \mathrm{~m}^{-2}$ with relatively low variability (Table 3). Higher values are obtained for flax:PVA with 30 wt. $\%$ PVA bonder fiber with surface mass values of about $465 \mathrm{~g} \mathrm{~m}^{-2}$. This is typical variability of nonwovens due to random deposition of fibers during nonwoven formation. In general terms, dispersion is relatively low between 4-7\%. With regard to nonwovens with bicomponent PA6/CoPA binder fiber, the surface mass is similar for different PA6/CoPA contents as observed in Table 3. Surface mass values are in a 
narrow range between $415-427 \mathrm{~g} \mathrm{~m}^{-2}$ with low standard deviation which represents a percentage variation of less than $3.5 \%$.

\section{Table 3}

Thickness of flax:PVA nonwovens is in the 1.5-2.0 $\mathrm{mm}$ range as observed in Table 4. Nevertheless we observe relatively high dispersion values (between $10-16 \%$ ) due to the nature of the deposition process on the hydroformer station. Regarding to flax:PA6/CoPA nonwovens, the average thickness is close to $2 \mathrm{~mm}$ with lower variability. In both cases, an average thickness of $2 \mathrm{~mm}$ can be achieved with the wetlaid process.

\section{Table 4}

Once surface mass and thickness values have been described, we proceed with mechanical properties of flax nonwovens thermally bonded with PVA and PA6/CoPA binding fibers. In the case of flax:PVA nonwovens, the previous SEM study has revealed low interaction between flax and melted PVA fibers. Thus, relatively poor mechanical resistance can be expected. Fig. 3 shows a comparative plot of the longitudinal tensile strength of flax nonwovens with PVA and PA6/CoPA binding fibers. With regard to flax:PVA, we can see that tensile strength is relatively low with values below $100 \mathrm{~N}$. Also, as the PVA binder fiber increases, the tensile strength even decreases thus indicating poor interaction after the thermal-bonding process $(91,72$ and $61 \mathrm{~N}$ for 10, 20 and 30 wt. \% PVA binder fiber respectively). These results are in total agreement with microstructure studied by SEM which has revealed absence of melted 
PVA fibers so that, flax fibers can't be fully embedded by PVA polymer. With regard to flax nonwovens thermally bonded with PA6/CoPA bicomponent fiber, tensile strength is highly dependent on total content of binding fiber. Therefore, the tensile strength changes from values around $174 \mathrm{~N}$ for $10 \mathrm{wt} \% \%$ PA6/CoPA fiber (real binder content of 5 wt. \% CoPA) up to values of about $489 \mathrm{~N}$ for $30 \mathrm{wt}$ \% PA6/CoPA fiber (real binder content of 15 wt. \% CoPA polymer). Nevertheless, it is important to remark that the only binding component is the CoPA sheath with a melting point at $135^{\circ} \mathrm{C}$ while the PA6 core fiber remains solid and contributes to improve mechanical performance of nonwovens with flax.

\section{Figure 3}

As the hydroformer station is characterized by a preferential nonwoven formation direction, the transversal mechanical response has also been evaluated. Fig. 4 shows a comparative plot of the tensile strength (transversal direction) for flax nonwovens with PVA and PA6/CoPA binder fibers. In the case of flax:PVA nonwovens, we observe similar tendency than observed for longitudinal direction but even lower values are obtained so that indicating that better mechanical properties are obtained in the preferential formation direction. The tensile strength is lowet than $72 \mathrm{~N}$ and an increase in PVA binder content doesn't lead to improvement on mechanical response due to the lack of interaction between PVA and flax fibers. Nevertheless, when using PA6/CoPA bicomponent fiber, we observe a clear increasing tendency on tensile strength values as the binder content increases. Nonwovens with 10 wt. \% PA6/CoPA (5 wt. \% CoPA binder) show a transversal tensile strength of $145 \mathrm{~N}$ and this is increased up to $341 \mathrm{~N}$ for nonwovens with $30 \mathrm{wt}$ \% PA6/CoPA fiber (15 wt. \% CoPA binder). If 
we compare transversal mechanical response of flax:PA6/CoPA to longitudinal response, we also corroborate that the longitudinal (preferential) direction provides better mechanical properties since the intrinsic nature of the hydroformer promotes slight higher fiber alignement on longitudinal direction. Once again, these results are in total agreement with those predicted by SEM study since flax:PA6/CoPA nonwovens are characterized by presence of low melt point CoPA polymer which melts during drying and subsequent calendering to act as a bridge between flax fibers and flax-PA6 core fibers and these bridges act as interlock points; so that, as the total content on CoPA binder increases, mechanical performace is improved.

\section{Figure 4}

With regard to elongation, Fig. 5 and Fig. 6 show a plot comparison between elongation at break values for flax nonwovens with different binder fibers for longitudinal (Fig. 5) and transversal (Fig. 6) directions. In general terms, elongation at break is representative for nonwoven cohesion. We can clearly observe that flax:PVA nonwovens show relatively low elongation at break values, around $2 \%$ for both longitudinal and transversal directions while elongation at break of flax:PA6/CoPA nonwovens is located around 5\%. This is in accordance with the SEM study. Flax:PVA nonwovens are characterized by absence of interlock points since PVA doesn't melt appropriately during the drying-calendering stage, so that when subjected to tensile stress, the overall cohesion can be easily lost with relatively low elongation at break values. On other hand the SEM study has revealed the melt of CoPA sheath thus allowing flax-flax and flax-PA6 interactions which lead to high cohesion on flax:PA6/CoPA nonwovens; therefore, higher elongation at break values are needed in 
order to break the highly crosslinked structure. In the case of elongation at break we do not observe differences between longitudinal and transversal directions and furthermore, we don't see differences in terms of the total binder content, so we can conclude that elongation at break is not a sensitive property to total binder content.

\section{Figure 5}

\section{Figure 6}

\subsection{Thermal and acoustic insulation properties of flax nonwovens thermally-bonded with different thermoplastic fibers.}

The increasing use of nonwovens in technical applications requires, in most cases, good thermal and acoustic insulation properties for comfort. For this reason, both thermal and acoustic insulation behavior of flax:PVA and flax:PA6/CoPA nonwovens has been evaluated. With regard to acoustic insulation, as the total thickness of nonwovens is relatively low (in the $1.5-2.0 \mathrm{~mm}$ range), the acoustic insulation properties of twelve stacked substrates has been evaluated in a Kundt's tube. Fig. 7 shows a plot evolution of the acoustic absorption coefficient in terms of the sound frequency for flax:PVA and flax:PA6/CoPA nonwovens. We can see that acoustic absorption at low frequencies (below $300 \mathrm{~Hz}$ ) is very low, with absorption coefficients in the $0.05-0.2$ range; in this frequency range, flax:PA6/CoPA show slightly higher acoustic insulation properties than flax:PVA nonwovens, but differences are not significant. In the medium frequencies range we can observe some interesting differences. With regard to flax:PVA nonwovens, the absorption coefficient increases up to values of about $0.4-0.5$ in the frequencies range comprised between $300 \mathrm{~Hz}-2$ $\mathrm{kHz}$, thus indicating interesting and quite homogenous acoustic insulation properties in 
this range. Regarding to flax:PA6/CoPA nonwovens, we observe a remarkable increase in absorption coefficient up to almost 0.6 at about $500-600 \mathrm{~Hz}$, but it decreases up to values around $0.2-0.3$ in the $1-2 \mathrm{kHz}$ range. In the high frequencies range (over $2 \mathrm{kHz}$ ), once again flax:PVA nonwovens show higher acoustic insulation properties than flax:PA6/CoPA. In general terms we observe good acoustic insulation properties for both flax:PVA and flax:PA6/CoPA nonwovens in the medium frequencies range; therefore these nonwoven structures show attracting properties for technical applications as acoustic absorbers.

\section{Figure 7}

In addition to acoustic insulation properties, thermal conductivity has also been determined by the heat flow meter method. Table 5 shows summarized results of thermal conductivity and thermal resistance for different contents on PVA and PA6/CoPA binder fibers. Thermal conductivity of flax:PVA nonwovens is relatively low, as expected, due to the high thermal insulation properties of flax fibers. Specifically, thermal conductivity is lower than $0.024 \mathrm{~W}(\mathrm{~m} \mathrm{~K})^{-1}$ for all flax:PVA compositions considered in this work. This fact is interesting since a thermal insulating material is characterized by thermal conductivity values lower than $0.060 \mathrm{~W}(\mathrm{~m} \mathrm{~K})^{-1}$; therefore, flax:PVA fulfill these requierements. Despite this, a thermal insulating material is also characterized by a thermal resistance higher than $0.25 \mathrm{~m}^{2} \mathrm{~K} \mathrm{~W}^{-1}$. So that, as thermal resistance values change in the $0.060-0.075 \mathrm{~m}^{2} \mathrm{~K} \mathrm{~W}^{-1}$, this means that a minimum of 3-4 stacked flax:PVA nonwovens would be necessary to fully consider this material as a thermal insulating component. With regard to flax:PA6/CoPA nonwovens, the thermal conductivity is higher with values in the $0.090-0.109 \mathrm{~W}$ (m 
$\mathrm{K})^{-1}$. Regarding to thermal resistance, values ranging from 0.012 to $0.018 \mathrm{~m}^{2} \mathrm{~K} \mathrm{~W}^{-1}$ are obtained and this means that a minimum of 15-20 stacked nonwoven substrates must be used to obtain fully thermal insulating behavior. So that, we can conclude that thermal insulating properties of flax:PVA nonwovens are better that flax:PA6/CoPA materials.

\section{Table 5}

\section{Conclusions.}

The use of the wet-laid technique to obtain flax-based nonwovens with different binder thermoplastic fibers has been validated. Thermo-bonding process with PVA binder fibers at $195^{\circ} \mathrm{C}$ leads to partially bonded flax:PVA nonwovens as observed by SEM study, since this temperature is not enough to provide full melting but the use of higher temperatures could lead to PVA degradation. This fact is responsible for mechanical performance of these nonwovens characterized by almost constant tensile strength values independently of the PVA binder fiber content (in the $10-30$ wt. \% range). The use of bicomponent PA6/CoPA binder fibers with PA6 core with a melt point of $222^{\circ} \mathrm{C}$ and a CoPA sheath with a low melting point located at $135^{\circ} \mathrm{C}$, allows full sheath melting and therefore, flax fibers can be embedded by melted CoPA polymer. This allows flax-flax and flax-PA6 interactions which are responsible for mechanical performance enhancement. In this case, a remarkable increase in tensile strength with the total content of PA6/CoPA binder fiber has been observed. On other hand, as the hydroformer station is characterized by a preferential deposition direction, some anisotropy on nonwovens has been detected since tensile strength values are higher in the longitudinal (preferential) direction than transversal direction. 
Although flax:PVA nonwovens show lower mechanical performance than flax:PA6/CoPA substrates, the acoustical insulation properties (measured through the absorption coefficient) are better and more homogeneous for flax:PVA nonwovens in the middle frequencies range. With regard to thermal insulating properties, both flax:PVA and flax:PA6/CoPA nonwovens offer interesting properties but once again, flax:PVA nonwovens possess lower thermal conductivity and good thermal resistance.

In general terms we can conclude that the wet-laid technique is useful to obtain nonwovens from natural flax fibres with different binder thermoplastic fibers. Although some anisotropy is detected, due to intrinsic preferential deposition direction, mechanical performance is enough to ensure good handling. In addition to this, interesting acoustic and thermal insulation properties can be obtained by stacking different sheets thus allowing the use of these materials as technical substrates for sound absorption or thermal insulation applications.

\section{Acknowledgements}

This work is part of the project IPT-310000-2010-037,"ECOTEXCOMP: Research and development of textile structures useful as reinforcement of composite materials with marked ecological character" funded by the "Ministerio de Ciencia e Innovacion", with an aid of 189540.20 euros, within the "Plan Nacional de Investigación Científica, Desarrollo e InnovaciónTecnológica 2008-2011" and funded by the European Union through FEDER funds, Technology Fund 2007-2013, Operational Programme on $\mathrm{R}+\mathrm{D}+\mathrm{i}$ for and on behalf of the companies." It is also acknowledged the project "WET-TEX: Implementación de la tecnología wet-laid en el desarrollo de nuevos textiles médico-sanitario" with expedient number IMIDIC/2010/137 (total aid of 284400 euro), and the project "WET-TEX II: 
Implementación de la tecnología wet-laid en la investigación y desarrollo de paneles para aplicaciones técnicas a partir de resíduos procedentes de la industria textil." with expedient number IMDEEA/2011/167 (total aid of 255000 euro) funded by IMPIVA and cofunded $(80 \%)$ by the European Union through FEDER funds, Valencian Community Operational 2007-2012. 


\section{References}

1. Chen JJ and Guo Z. The Acoustic Insulation Property of a New Non-Woven Material. Advanced Engineering Materials. 2011; 194-196: 471-475.

2. Parikh DV, Chen Y, Sachinvala ND, et al. Sound dampening by velour nonwoven systems in automobiles. AATCC Rev. 2006; 6: 40-44.

3. Debnath $\mathrm{S}$ and Madhusoothanan M. Thermal insulation, compression and air permeability of polyester needle-punched nonwoven. Indian J. Fibre Text. Tes. 2010; 35: 38-44.

4. Lin CM, Lou CW, and Lin JH. Manufacturing and Properties of Fire-Retardant and Thermal Insulation Nonwoven Fabrics with FR-Polyester Hollow Fibers. Text. Res. J. 2009; 79: 993-1000.

5. Lou CW, Lin CM, Huang CC, et al. Manufacturing and physical properties of fire-retardant fibrous laminate thermal insulation. Fiber. Polym. 2008; 9: 431437.

6. Tai KC, Chen P, Lin CW, et al., Evaluation on the Sound Absorption and Mechanical Property of the Multi-layer Needle-punching Nonwoven, in MultiFunctional Materials and Structures Iii, Pts 1 and 2. 2010. p. 475-478.

7. Parikh DV, Chen Y, and Sun L. Reducing automotive interior noise with natural fiber nonwoven floor covering systems. Text. Res. J. 2006; 76: 813-820.

8. Neira DSM and Marinho GS. Nonwoven Sisal Fiber as Thermal Insulator Material. J. Nat. Fibers. 2009; 6: 115-126.

9. Parikh DV, Sachinvala ND, Chen Y, et al. Acoustic properties of environmentally benign automotive natural fiber composites. AATCC Rev. 2006; 6: 43-48. 
10. Reddy N and Yang YQ. Light-Weight Polypropylene Composites Reinforced with Whole Chicken Feathers. J. Appl. Polym. Sci. 2010; 116: 3668-3675.

11. Harwood J, McCormick P, Waldron D, et al. Evaluation of flax accessions for high value textile end uses. Ind. Crop. Prod. 2008; 27: 22-28.

12. Soukupova V, Boguslavsky L, and Anandjiwala RD. Studies on the properties of biodegradable wipes made by the hydroentanglement bonding technique. Text. Res. J. 2007; 77: 301-311.

13. Dobircau L, Sreekumar PA, Saiah R, et al. Wheat flour thermoplastic matrix reinforced by waste cotton fibre: Agro-green-composites. Compos. Pt. A-Appl. Sci. Manuf. 2009; 40: 329-334.

14. Marshall WE, Akin DE, Wartelle LH, et al. Citric acid treatment of flax, cotton and blended nonwoven mats for copper ion absorption. Ind. Crop. Prod. 2007; 26: 8-13.

15. El Hajj N, Mboumba-Mamboundou B, Dheilly RM, et al. Development of thermal insulating and sound absorbing agro-sourced materials from auto linked flax-tows. Ind. Crop. Prod. 2011; 34: 921-928.

16. Lee BO, Ko JA, and Han SW, Characteristics of PP/PET Bicomponent Melt Blown Nonwovens as Sound Absorbing Material, in Multi-Functional Materials and Structures Iii, Pts 1 and 2. 2010. p. 935-938.

17. Tascan M, Vaughn EA, Stevens KA, et al. Effects of total surface area and fabric density on the acoustical behavior of traditional thermal-bonded highloft nonwoven fabrics. J. Text. Inst. 2011; 102: 746-751.

18. Vigneswaran C, Chandrasekaran K, and Senthilkumar P. Effect of Thermal Conductivity Behavior of Jute/Cotton Blended Knitted Fabrics. J. Ind. Text. 2009; 38: 289-307. 
19. Lou CW, Lin CW, Lin CC, et al., The Effects of Thermal Consolidation Methods on PET Nonwoven Composites for Thermal Insulation Use, in Smart Materials, T. Tunkasiri, Editor. 2008. p. 405-408.

20. Anantharamaiah N, Verenich S, and Pourdeyhimi B. Durable Nonwoven Fabrics via Fracturing Bicomponent Islands-in-the-Sea Filaments. AATCC Rev. 2009; 9: $41-47$.

21. Pillin I, Kervoelen A, Bourmaud A, et al. Could oleaginous flax fibers be used as reinforcement for polymers? Ind. Crop. Prod. 2011; 34: 1556-1563.

22. Parikh DV, Calamari TA, Sawhney APS, et al. Thermoformable automotive composites containing kenaf and other cellulosic fibers. Text. Res. J. 2002; 72: 668-672.

23. Anandjiwala RD and Boguslavsky L. Development of needle-punched nonwoven fabrics from flax fibers for air filtration applications. Text. Res. J. 2008; 78: 614-624.

24. Patanaik A and Anandjiwala R. Some Studies on Water Permeability of Nonwoven Fabrics. Text. Res. J. 2009; 79: 147-153.

25. Yoon MJ, Doh SJ, and Im JN. Preparation and Characterization of Carboxymethyl Cellulose Nonwovens by a Wet-laid Process. Fiber. Polym. 2011; 12: 247-251.

26. Fedorova N, Verenich S, and Pourdeyhimi B. Strength Optimization of Thermally Bonded Spunbond Nonwovens. J. Eng. Fiber Fabr. 2007; 2: 38-48.

27. Kim HS, Ito H, Kikutani T, et al. Computational analysis on the thermal bonding behaviour of bicomponent fibres. J. Text. Inst. 1999; 90: 508-525. 
28. Rawal A, Priyadarshi A, Lomov SV, et al. Tensile behaviour of thermally bonded nonwoven structures: model description. J. Mater. Sci. 2010; 45: 22742284.

29. Safavi A, Fathi S, Babaei MR, et al. Experimental and numerical analysis of fiber characteristics effects on fiber dispersion for wet-laid nonwoven. Fiber. Polym. 2009; 10: 231-236. 


\section{Figure legends}

Scheme 1.- General scheme of the wet-laid process with a water-fiber dispersión and water recovery system.

Figure 1.- SEM images of flax nonwovens thermally bonded with PVA in different flax:PVA wt. \% ratio and different magnification. a) 90:10, x200; b) 90:10, x400; c) $80: 20, x 200 ;$ d) 80:20, x400; e) 70:30, x200; f) 70:30, x400.

Figure 2.- SEM images of flax nonwovens thermally bonded with PA6/CoPA in different flax:PA6/CoPA wt. \% ratio and different magnification. a) 90:10, x200; b) $90: 10, x 400$; ) 80:20, x200; d) 80:20, x400; e) 70:30, x200; f) 70:30, x400.

Figure 3.- Longitudinal tensile strength of flax nonwovens thermally-bonded with PVA and PA6/CoPA fibers in terms of the wt. \% of the binding fiber.

Figure 4.- Transversal tensile strength of flax nonwovens thermally-bonded with PVA and PA6/CoPA fibers in terms of the wt. $\%$ of the binding fiber.

Figure 5.- Longitudinal elongation at break of flax nonwovens thermally-bonded with PVA and PA6/CoPA fibers in terms of the wt. $\%$ of the binding fiber.

Figure 6.- Transversal elongation at break of flax nonwovens thermally-bonded with PVA and PA6/CoPA fibers in terms of the wt. \% of the binding fiber.

Figure 7.- Plot evolution of the absorption coefficient of flax nonwovens thermallybonded with PVA and PA6/CoPA fibers (flax:binder wt. ratio $=80: 20$ ) in terms of the frequency. 


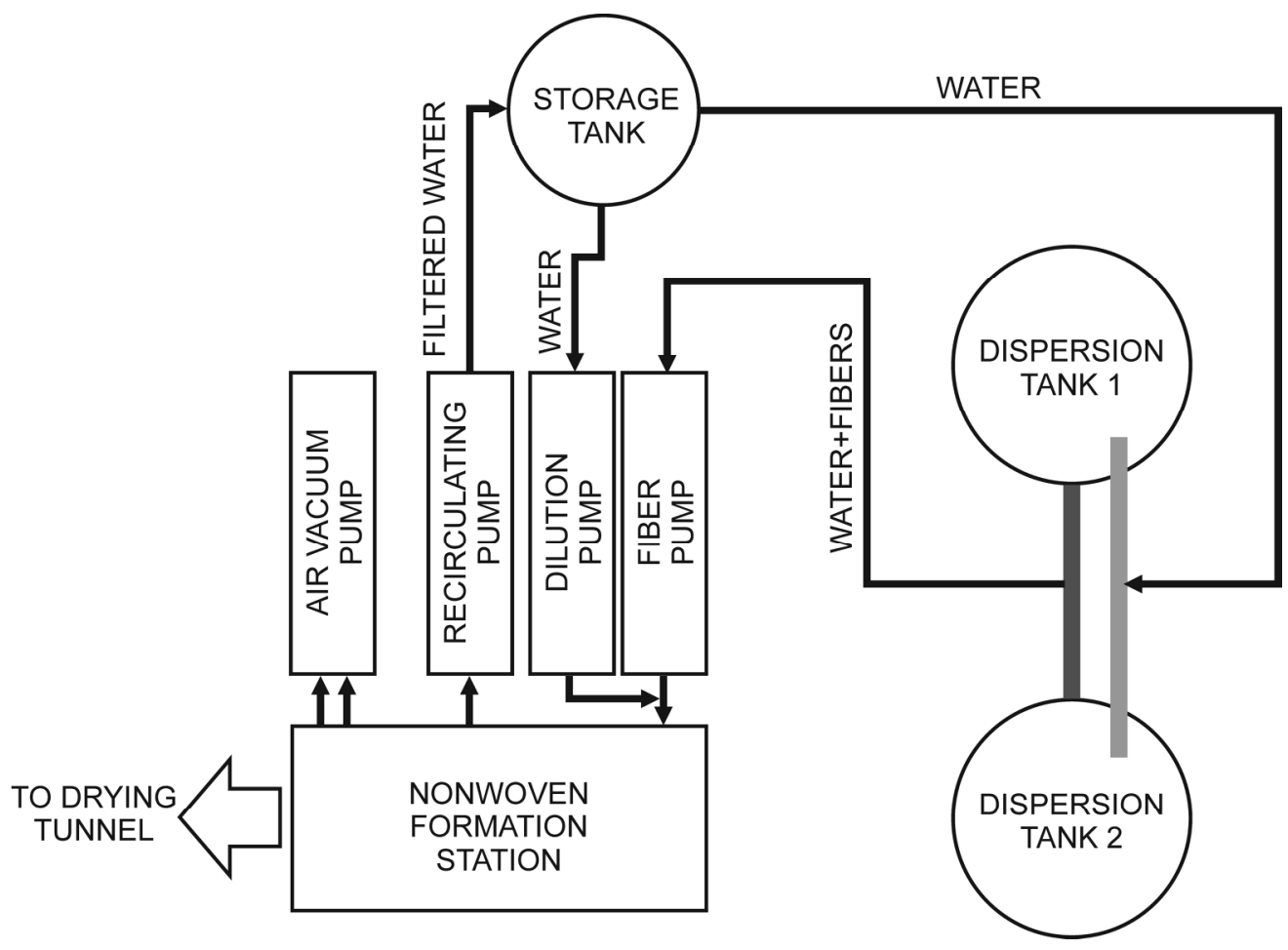

Scheme 1.- General scheme of the wet-laid process with a water-fiber dispersión and water recovery system. $108 \times 78 \mathrm{~mm}(600 \times 600 \mathrm{DPI})$ 

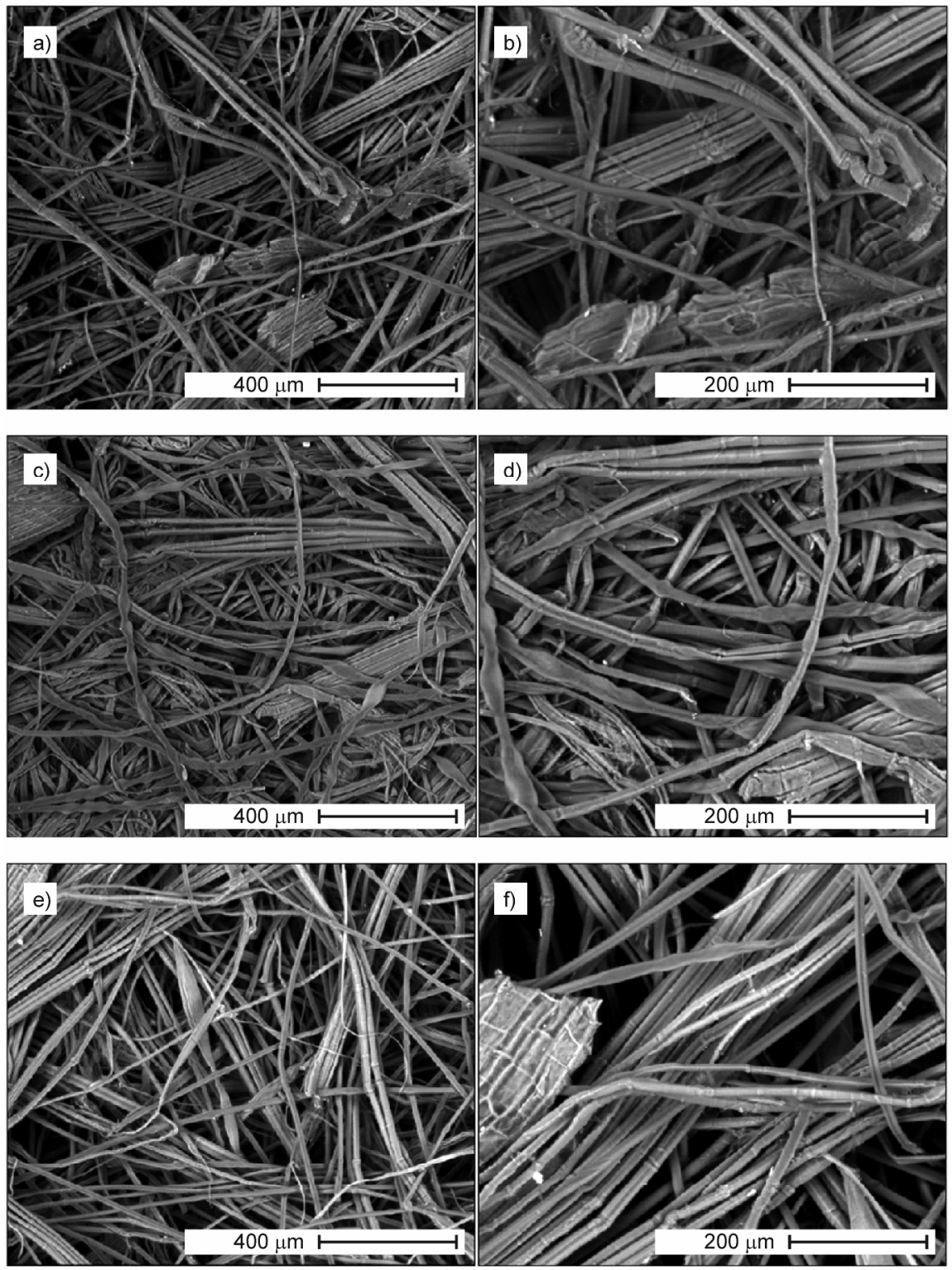

Figure 1.- SEM images of flax nonwovens thermally bonded with PVA in different flax:PVA wt. \% ratio and different magnification. a) $90: 10$, x200; b) $90: 10$, x400; c) $80: 20$, x200; d) $80: 20$, x400; e) 70:30, x200; f) $70: 30, x 400$.

$260 \times 348 \mathrm{~mm}(300 \times 300 \mathrm{DPI})$ 

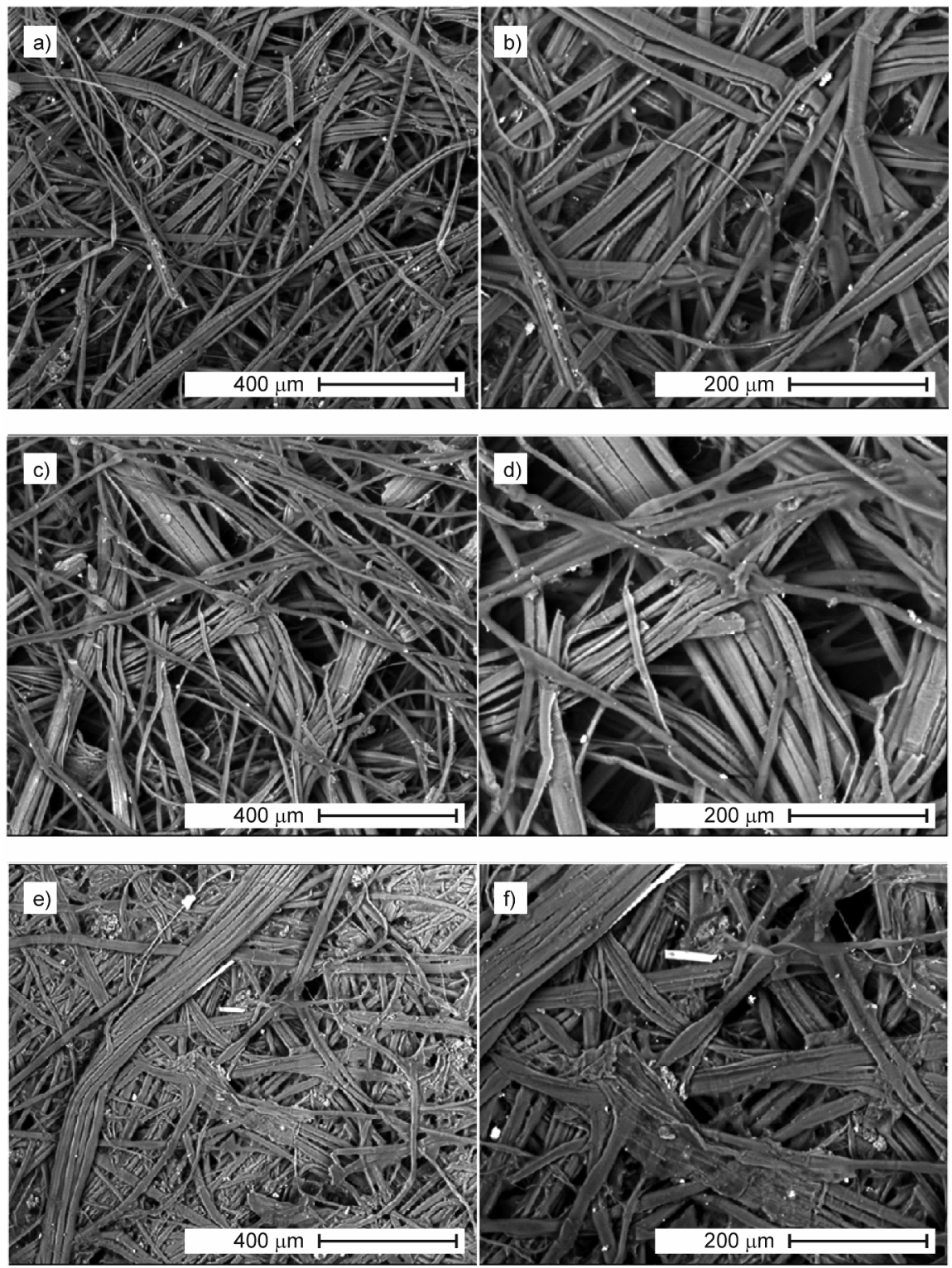

Figure 2.- SEM images of flax nonwovens thermally bonded with PA6/CoPA in different flax:PA6/CoPA wt. \% ratio and different magnification. a) 90:10, x200; b) 90:10, x400; c) 80:20, x200; d) 80:20, x400; e) $70: 30, x 200 ;$ f) $70: 30, x 400$. $260 \times 348 \mathrm{~mm}(300 \times 300 \mathrm{DPI})$ 
Figure 3.- Longitudinal tensile strength of flax nonwovens thermally-bonded with PVA and PA6/CoPA fibers in terms of the wt. \% of the binding fiber.

$53 \times 37 \mathrm{~mm}(600 \times 600 \mathrm{DPI})$ 
Figure 4.- Transversal tensile strength of flax nonwovens thermally-bonded with PVA and PA6/CoPA fibers in terms of the wt. \% of the binding fiber. $53 \times 37 \mathrm{~mm}(600 \times 600 \mathrm{DPI})$

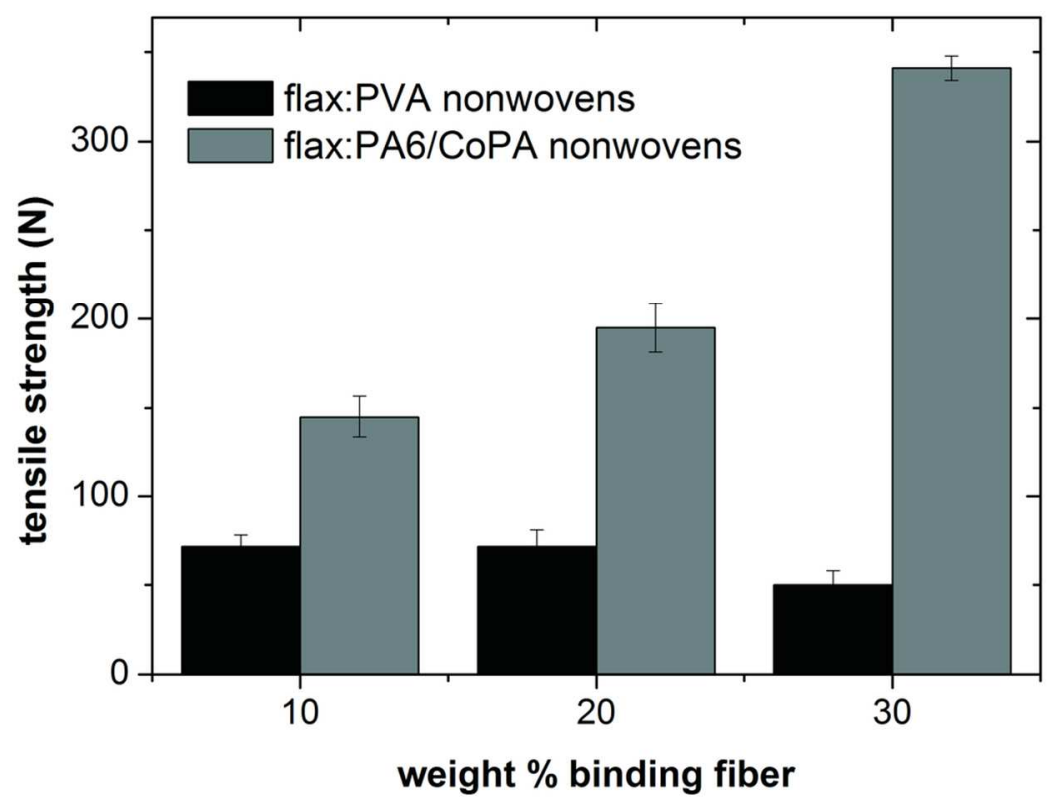


Figure 5.- Longitudinal elongation at break of flax nonwovens thermally-bonded with PVA and PA6/CoPA fibers in terms of the wt. \% of the binding fiber. $53 \times 37 \mathrm{~mm}(600 \times 600$ DPI $)$ 
Figure 6.- Transversal elongation at break of flax nonwovens thermally-bonded with PVA and PA6/CoPA fibers in terms of the wt. \% of the binding fiber. $53 \times 37 \mathrm{~mm}(600 \times 600 \mathrm{DPI})$ 


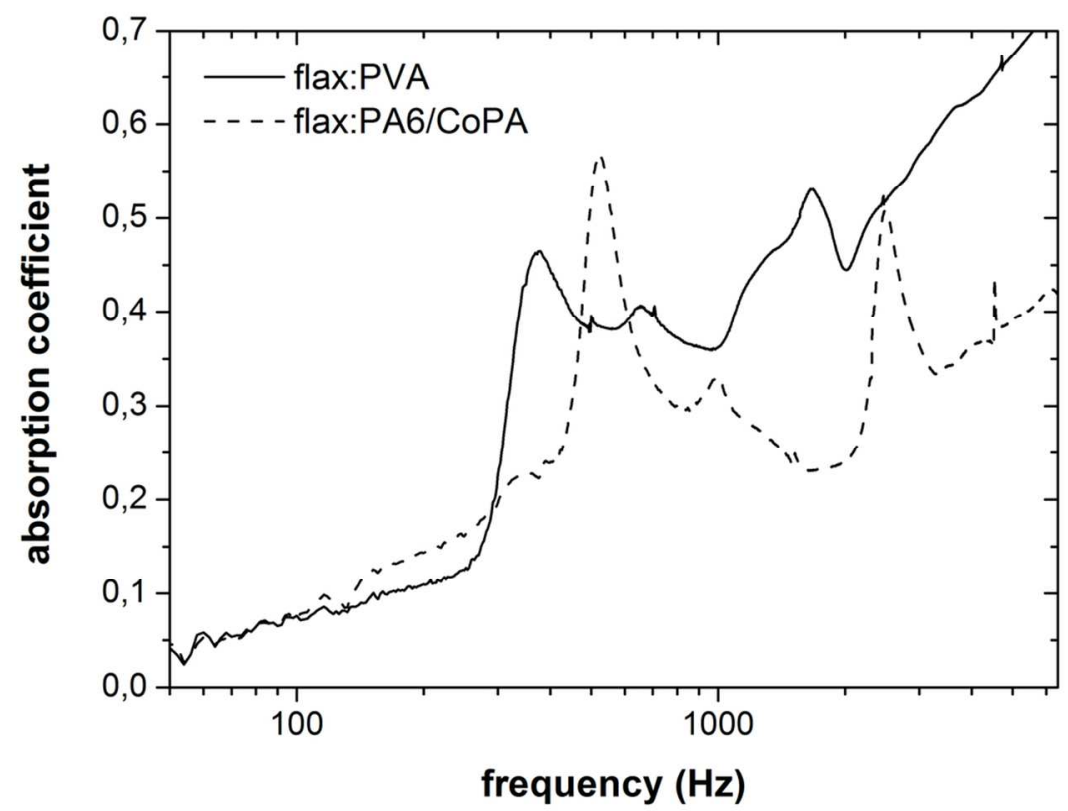

Figure 7.- Plot evolution of the absorption coefficient of flax nonwovens thermally-bonded with PVA and PA6/CoPA fibers (flax:binder wt. ratio $=80: 20$ ) in terms of the frequency. $53 \times 37 \mathrm{~mm}(600 \times 600 \mathrm{DPI})$ 
Table 1.- General characteristics of flax fibers used as base fibers for nonwoven formation by wet-laid process on a hydroformer station.

\begin{tabular}{|l|l|}
\hline Transversal section & Polygonal with oval \\
\hline Thickness $[\mu \mathrm{m}]$ & $10-500$ \\
\hline Density $\left[\mathrm{g} \mathrm{cm}^{-3}\right]$ & $1.4-1.5$ \\
\hline Resistance to constant heat $\left[{ }^{\circ} \mathrm{C}\right]$ & $75-80$ \\
\hline Decomposition temperature $\left[{ }^{\circ} \mathrm{C}\right]$ & 300 \\
\hline Moisture content $[\%]$ & 15 \\
\hline Color & Natural \\
\hline Fiber length [mm] & 6 \\
\hline Light resistance & Moderate \\
\hline Resistance to acids & Moderate \\
\hline Resistance to alkalis & Moderate \\
\hline Water swelling & Moderate \\
\hline
\end{tabular}


Table 2.- Processing parameters in the drying and calendering stages for flax nonwovens thermally bonded with PVA and PA6/CoPA thermoplastic fibers.

\begin{tabular}{|l|l|l|}
\hline \multirow{4}{*}{ Drying stage } & Temperature at the drying module $\left({ }^{\circ} \mathrm{C}\right)$ & 195 \\
\cline { 2 - 3 } & Temperature at the boiler $\left({ }^{\circ} \mathrm{C}\right)$ & 250 \\
\cline { 2 - 3 } Calendering stage & Drying time (min) & 15 \\
\hline & Distance between calendering rollers $(\mathrm{mm})$ & 0 \\
\cline { 2 - 3 } & Pressure (bar) & 40 \\
\cline { 2 - 3 } & Temperature at the roller surface $\left({ }^{\circ} \mathrm{C}\right)$ & 200 \\
\cline { 2 - 3 } & Linear pressure on nonwoven $\left(\mathrm{N} \mathrm{mm}{ }^{-1}\right)$ & 124 \\
\hline
\end{tabular}


Table 3.- Average surface mass values of flax nonwovens thermally bonded with PVA and PA6/CoPA thermoplastic fibers.

\begin{tabular}{|l|c|c|c|c|}
\hline \multirow{2}{*}{$\begin{array}{l}\text { Weight \% } \\
\text { flax:binder }\end{array}$} & \multicolumn{2}{|c|}{ flax:PVA fiber nonwovens } & \multicolumn{2}{l|}{ flax: PA6/COPA fiber nonwovens } \\
\cline { 2 - 5 } & Average & Standard deviation & Average & Standard deviation \\
\hline $90 / 10$ & 394.0 & 16.8 & 415.4 & 14.4 \\
\hline $80 / 20$ & 404.2 & 23.8 & 419.0 & 8.6 \\
\hline $70 / 30$ & 465.4 & 35.1 & 426.8 & 9.0 \\
\hline
\end{tabular}

20

21

22 
Table 4.- Average thickness values of flax nonwovens thermally bonded with different thermoplastic fibers.

\begin{tabular}{|l|c|c|c|c|}
\hline \multirow{2}{*}{$\begin{array}{l}\text { Weight \% } \\
\text { flax:binder }\end{array}$} & \multicolumn{2}{|c|}{ flax:PVA fiber nonwovens } & \multicolumn{2}{c|}{ flax: PA6/COPA fiber nonwovens } \\
\cline { 2 - 5 } & Average & Standard deviation & Average & Standard deviation \\
\hline $90: 10$ & 1.52 & 0.25 & 2.15 & 0.23 \\
\hline $80: 20$ & 1.94 & 0.09 & 2.17 & 0.12 \\
\hline $70: 30$ & 1.43 & 0.14 & 1.96 & 0.07 \\
\hline
\end{tabular}


Table 5.- Thermal insulation properties of flax:PVA and flax:PA6/CoPA nonwovens in terms of the binder fiber content.

\begin{tabular}{|c|c|c|c|c|}
\hline \multirow[b]{2}{*}{$\begin{array}{l}\text { Weight \% } \\
\text { flax:binder }\end{array}$} & \multicolumn{2}{|c|}{ flax:PVA fiber nonwovens } & \multicolumn{2}{|c|}{ flax: PA6/COPA fiber nonwovens } \\
\hline & $\begin{array}{c}\text { Thermal } \\
\text { conductivity } \\
{\left[\mathrm{W}(\mathrm{m} \mathrm{K})^{-1}\right]}\end{array}$ & $\begin{array}{l}\text { Thermal } \\
\text { resistance } \\
\left(\mathrm{m}^{2} \mathrm{~K} \mathrm{~W}^{-1}\right)\end{array}$ & $\begin{array}{c}\text { Thermal } \\
\text { conductivity } \\
{\left[\mathrm{W}(\mathrm{m} \mathrm{K})^{-1}\right]}\end{array}$ & $\begin{array}{l}\text { Thermal } \\
\text { resistance } \\
\left(\mathrm{m}^{2} \mathrm{~K} \mathrm{~W}^{-1}\right)\end{array}$ \\
\hline $90 / 10$ & 0.020 & 0.065 & 0.093 & 0.018 \\
\hline $80 / 20$ & 0.024 & 0.075 & 0.109 & 0.012 \\
\hline $70 / 30$ & 0.023 & 0.060 & 0.090 & 0.016 \\
\hline
\end{tabular}

